\title{
Focused Helium Ion Beam Nanomachining of Thin Membranes vs. Bulk Substrates
}

\author{
E.M. Mutunga, ${ }^{1}$ S. Lockerman, ${ }^{1}$ S. Tan, ${ }^{2}$ R. Livengood, ${ }^{2}$ A.E. Vladár, ${ }^{3}$ and K.L. Klein, ${ }^{1,3 *}$ \\ ${ }^{1}$ University of the District of Columbia, 4200 Connecticut Ave NW, Washington, DC 20008 \\ ${ }^{2}$ Intel Corporation, Santa Clara, CA, 95054 \\ ${ }^{3}$ National Institute of Standards and Technology, 100 Bureau Drive, Gaithersburg, MD 20899 \\ ${ }^{*}$ Corresponding author: kate.klein@udc.edu
}

The helium ion microscope (HIM) beam has a characteristically low sputter yield and smaller interaction volume near the surface as compared to focused gallium ion beam [1]. As a result, nanometer-scale structures with feature sizes of $5 \mathrm{~nm}$ or smaller have been machined in thin membranes, for applications such as nanopore sensors and optical devices [2-4]. Still, inconsistent results have been observed in bulk substrates where the region often appears to swell rather than mill [5]. This study seeks to understand how mill rates and beam-substrate interaction profiles vary with substrate thickness and beam energy.

In recent work, we have compared 140-nm-thick membrane to bulk silicon interaction profiles through the use of TEM cross-sectional imaging, which illustrated that transmission milling (or forward sputtering) is quite significant [6]. In this paper we investigate the machining properties of gold and other relevant substrates using similar techniques. SRIM modeling [7] and mill rate experiments in thin films and bulk substrates were performed. Fig. 1 shows an example of a $200 \mathrm{~nm}$ box milled in a bulk substrate (a) as compared to a $100 \mathrm{~nm}$ gold foil (b) with comparable dose. The corresponding SRIM helium ion point trajectories at $30 \mathrm{keV}$ beam energy indicate that with an ion range of $\sim 87 \mathrm{~nm}$, a large portion (nearly $42 \%$ ) of ions are transmitted through the sample for a $100 \mathrm{~nm}$ substrate thicknesses; whereas in the bulk substrate all the incident helium ions, with the exception of backscattered ions $(\sim 19 \%)$, stop in the substrate. Achieving favorable nanomachining in bulk substrates is not straightforward due to the accumulation of dislocations and damage caused by the trapped helium in the surrounding and underlying areas. Therefore, it is optimal to use the lowest dose possible.

We further investigated the theoretical sputter yield as a function substrate thickness for various beam energies as shown in Fig 2. A decrease in backward sputter yield with beam energy is prevalent as a result of the shorter ion range for lower beam energies. For each beam energy the sputter yield reaches a constant rate at a thickness of approximately twice the ion range in bulk substrates, while at thicknesses below the range the sputter yield drops off significantly. It was also experimentally observed that for lower beam energies, the mill rate in a $100 \mathrm{~nm}$ gold foil increases. Fig. 3 compares the time it takes to completely mill a $200 \mathrm{~nm}$ box raster in $100 \mathrm{~nm}$ gold foil for beam energies of $20.5 \mathrm{keV}, 30 \mathrm{keV}$ and $35 \mathrm{keV}$. It was found that the experimentally-determined sputter yield for a $100 \mathrm{~nm}$ gold foil was significantly higher than theoretical calculations, presumably due to transmission milling $[6,8]$, which is not yet accounted for in the SRIM model.

[1] J.A. Notte, Microscopy Today 20, 16 (2012).

[2] L. Scipioni, et al., J. Vac. Sci. Technol. B 28, C6P18 (2010).

[3] J. Yang, et al., Nanotechnology 22, 285310, (2011).

[4] L. Scipioni, Carl Zeiss, Adv. Mat. Char.Workshop, U. Illinois, (2012).

[5] R. Livengood et al., J. Vac. Sci. Technol. B 27 (2009) 3244.

[6] S. Tan, K. Klein, D. Shima, R. Livengood, E. Mutunga, and A. Vladar, EIPBN proceedings (2014).

[7] J.F. Zeigler, M.D. Zeigler, and J.P. Biersack, SRIM-2012.03 modeling freeware.

[8] M.M. Marshall, J. Yang, and A.R. Hall, Scanning 34, 101 (2012). 


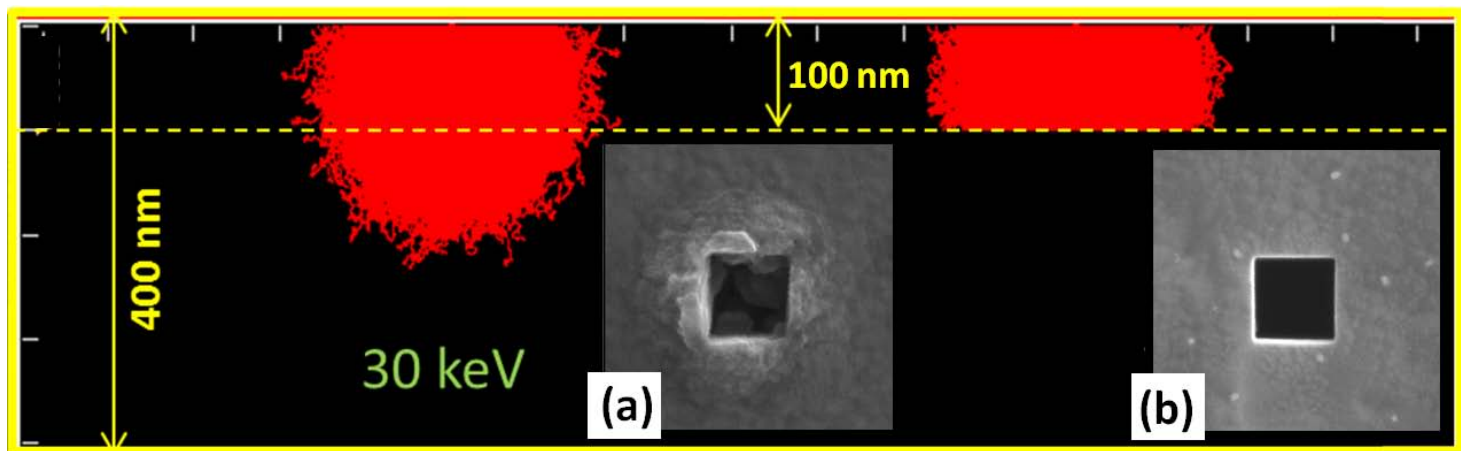

Figure 1. SRIM [6] helium ion point trajectories at $30 \mathrm{keV}$ beam energy for (a) $400 \mathrm{~nm}$ and (b) $100 \mathrm{~nm}$ gold substrate thicknesses with corresponding HIM images of a $200 \mathrm{~nm}$ box milled in (a) bulk substrate as compared to (b) 100-nm-thick gold foil (with comparable ion dose).

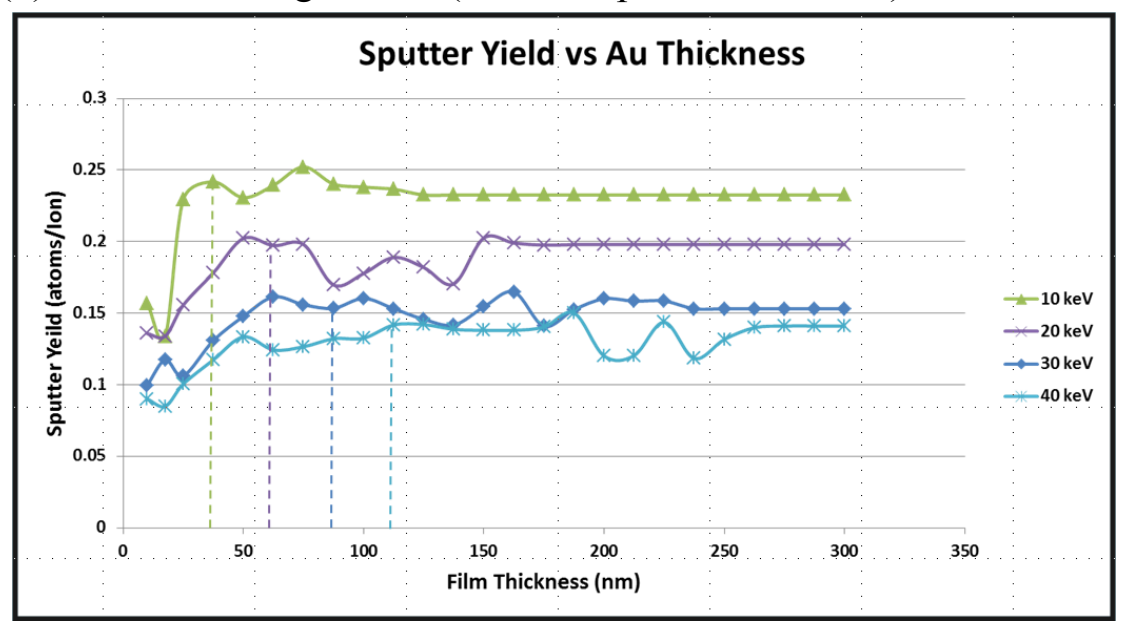

Figure 2. Theoretical sputter yield as a function substrate thickness for various beam energies. We note a decreased sputter yield with beam energy (transmission milling is neglected in this model). For each beam energy, the sputter yield reaches a constant rate at a thickness of approximately twice the ion range in bulk; at thicknesses below the bulk range (as indicated by the dashed lines), the sputter yield drops off significantly.

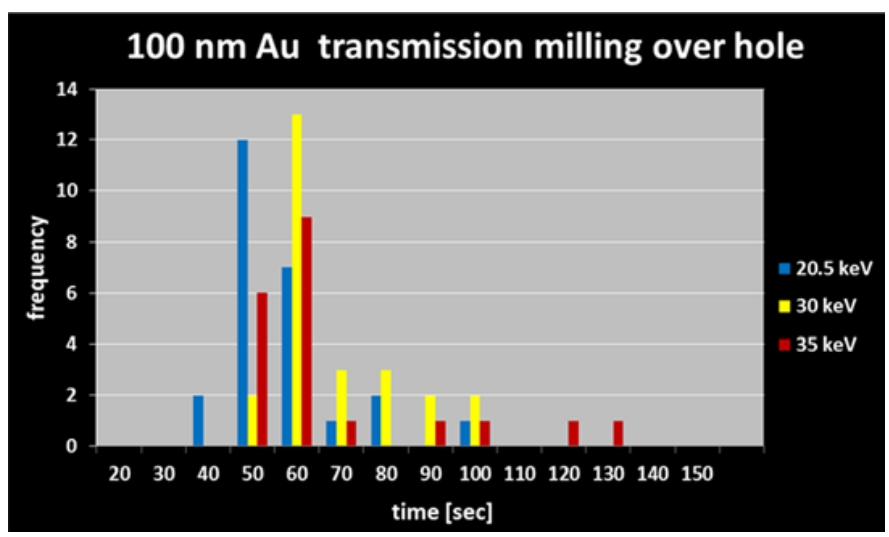

Figure 3. Comparison of experimental mill rates at $20.5 \mathrm{keV}, 30 \mathrm{keV}$ and $35 \mathrm{keV}$ beam energies for a $200 \mathrm{~nm}$ box raster to completely mill through a $100 \mathrm{~nm}$ gold foil, which demonstrates a trend towards improved sputter yield at lower beam energies. 